\title{
UK Nuclear Physics Threatened
}

Honouring European agreements figures among the implications of any implementatien of a proposal by the UK's Science and Engineering Research Council (SERC), announced last month, to achieve a "very significant" reduction (some 10\%) in government spending for nuclear and particle physics. The Council indicated that it will have to "make plans for a possible closure" of Daresbury Laboratory's Nuclear Structure Facility (NSF) if the SERC's Nuclear Physics Board (which handles both nuclear and particle physics) is to remain within budget. The proposal stemmed directly from a vote in Parliament to increase the SERC's $1991 / 92$ annual budget by $3 \%$ to $£ 451 \mathrm{M}$ (the major part of the $f 920 \mathrm{M}$ government allocation for research) at a time when cost inflation is around $10 \%$ per annum.

Commenting on the proposal, the Chairman of SERC Sir Mark Richmond said that Council would "look for ways" for a holding operation to run the facility through 1991 and 1992 to abide by the first stage of its EUROGAM agreement with the French CNRS for the construction of a new, ambulatory, $4 \pi$ detector for nuclear structure research. A study would be set up to assess the importance of the science involved to help Council in a "further consideration" of the issues.

The news that a possible immediate closure of the facility was being considered prompted a vigorous response by the Institute of Physics and the international physics community (some 570 letters of support were received). The IOP urged the Advisory Board of the Research Council (ABRC), the next higher authority, to release an additional $f 5 \mathrm{M}$ per year (it is understood that the SERC has agreed to place a high priority on such a bid). The IOP is also prepared to carry out an independent review. The Chairman of the Nuclear Physics Board, Professor A. Donnachie of Manchester University has indicated that the SERC panel's make-up and terms of reference, and by implication the time scale, will only become known as we go to press so one can only speculate on the futures of both studies.

The pressure on Daresbury partly reflects the generally low level of government support for basic research in the UK and a disproportionately small nuclear physics community. There are 200 permanent, fixed term and student nuclear physicists (roughly $15 \%$ and $20 \%$ the sizes of the French and German communities, respectively) with an annual budget of $£ 15 \mathrm{M}$ in 1987 as compared to an estimated $\mathrm{f} 85 \mathrm{M}$ in the former West Germany. The NSF with a staff of 130 (out of some 600 at Daresbury) thus represents a major part to the UK effort. Cutting it would have a major impact on nuclear physics in Britain and how it is perceived by young researchers at a time when the NSF is acclaimed, and indeed by the Chairman of the SERC, as "a leading facility for (nuclear structure) research in the world which we we hoped would keep the UK in the forefront ... for some years ahead" (the natural life of the NSF's 20 MV Tandem Van de Graaff accelerator has seven years to run).

The NSF's European engagements are also substantial. Dr. W. Gelletly, its Director, estimates that on average roughly $10 \%$ of the beam time of the accelerator is assigned to $50-60$ visitors from the Continent. This falls very much within the NSF's rôle as a user facility where only $6 \%$ of the work is for in-house research. Without access to this beam time, other European scientists may in the long run deny access to facilities by UK physicists, especially younger researchers, except on strictly business terms. The UK community would have to reckon on user charges in addition to travel costs, translating overall to $20 \%$ of today's beam time for $80 \%$ of the cost of the NSF.

The NSF also sees strengths in the area of instrumentation. The EUROGAM collaboration testifies to this, as does a special large solid angle, large acceptance angle detector called SUSAN that is being built to selectively detect the the products of quasi-elastic fragmentation and deep-inelastic nuclear reactions.

\section{Europe Concerned}

The Nuclear Structure Facility at Daresbury plays a leading rôle at the forefront of nuclear research. Its discoveries of superdeformation, the threshold anomaly and pseudospin symmetry constitute some of the central themes of modern nuclear physics that are defining directions for future work. So it is fair to say that the name Daresbury has become tantamount with that of new frontiers of nuclear structure research and of innovative technical developments.

This is why the SERC's announcement that it plans to close the NSF brought such a deep sense of dismay to the nuclear physics community. No previous warning of such a move had been issued, let alone the request for a peer review of the NSF's activities to help take such a serious decision. The move appeared to be solely the consequence of an unforeseen increase in the UK contribution to CERN that the SERC is committed to paying. SERC's Nuclear Physics Board, which funds both nuclear and particle physics, was therefore obliged to reduce significantly its support for nuclear structure physics.

The 570 letters (350 from foreign scientists) received by Sir Mark Richmond following the announcement were consistent in their message. But they were also very different in the way of expressing it so they clearly represented the views of individuals. UK nuclear physicists had themselves taken the campaign to Parliament with a debate focussing on funding for nuclear physics. The net result was a possible stay of execution until the end of 1992. The SERC has also reacted positively but one understands that with insufficient funds, it places a high priority on the bid to the ABRC. However, it is believed that the $A B R C$ is reluctant to
However, more extensive European-level collaboration on such projects following a possible a closure of the NSF would not solve the basic problem of access to beams. The NSF is therefore considering future options for beam activities. A source of beams of radioactive nuclei far from stability has been costed (one is presently confined to beams produced in fusion reactions and in radioactive decays). A project of this type has been proposed in Japan and the physics case for a US machine is presently being worked on.

A high current, proton spallation source (the optimum approach) may cost up to f100 M. Capitalising on the ISOLDE experience at CERN, it would use high energy protons from the SERC's Rutherford Appleton Laboratory's ISIS source to generate radioactive species by spallation. Low-energy radioactive ion beams obtained with an ISOLDE-type separator would then be reaccelerated. An alternative approach would employ an ion source and a cyclotron to feed Daresbury's tandem accelerator, thus providing a radioactive beam facility within an estimated three years for a relatively modest $\mathrm{f} 18 \mathrm{M}$.

provide additional finance so the closure of the NSF could still occur in 1991/92.

Given the situation it is impossible not to feel a mounting sense of rage against the architects of big science, not only because of the decision but also, and more particularly, because of their short-sightedness. An eminent high energy physicist recently gave the following assessment of nuclear and particle physics: ".... particle physicists are in search of simplicity ... (they) do not understand why other people (viz nuclear physicists) may like complicated systems".

What would Niels Bohr, the discoverer of the compound nucleus and a founder of CERN, have made of this? Nuclear physics with its connections to molecular and atomic physics, as well as to astrophysics and particle physics, has a unique rôle in the broad development of quantal physics. Scores of students testify to this, as does recent recognition by leading countries (ironically enough as a spin off of the outstanding work done at Daresbury's TESSA30 detector facility). Testaments to the new awareness are the funding of $4 \pi \gamma$-ray detectors (NORDBALL in Denmark, OSIRIS in Germany, GASP in Italy, GAMMASPHERE in the USA) and the planning for EUROBALL. The latter represents a European collaboration, for which EUROGAM is the initial phase, aimed at constructing the ultimate in $\gamma$-ray detectors.

So let us hope that common sense prevails and the NSF will be allowed to continue to play its leading rôle. Otherwise we shall have a perplexing display of poor judgment involving the closure of one of the most successful and powerful centres for nuclear research in Europe.

\section{R.A. Broglia, University of Milan}

Convener, NuPECC Nuclear Structure Group Member, EUROBALL Coordinating Committee 